\title{
REVIEW
}

\section{Clinical review: Early patient mobilization in the ICU}

\author{
Carol L Hodgson*1,2, Sue Berney ${ }^{3,4}$, Megan Harrold ${ }^{5,6}$, Manoj Saxena ${ }^{7,8,9}$ and Rinaldo Bellomo'
}

\begin{abstract}
Early mobilization (EM) of ICU patients is a physiologically logical intervention to attenuate critical illness-associated muscle weakness. However, its long-term value remains controversial. We performed a detailed analytical review of the literature using multiple relevant key terms in order to provide a comprehensive assessment of current knowledge on EM in critically ill patients. We found that the term EM remains undefined and encompasses a range of heterogeneous interventions that have been used alone or in combination. Nonetheless, several studies suggest that different forms of EM may be both safe and feasible in ICU patients, including those receiving mechanical ventilation. Unfortunately, these studies of EM are mostly single center in design, have limited external validity and have highly variable control treatments. In addition, new technology to facilitate EM such as cycle ergometry, transcutaneous electrical muscle stimulation and video therapy are increasingly being used to achieve such EM despite limited evidence of efficacy. We conclude that although preliminary low-level evidence suggests that EM in the ICU is safe, feasible and may yield clinical benefits, EM is also labor-intensive and requires appropriate staffing models and equipment. More research is thus required to identify current standard practice, optimal EM techniques and appropriate outcome measures before EM can be introduced into the routine care of critically ill patients.
\end{abstract}

\section{Introduction}

During critical illness, patients who are immobilized for more than a few days develop neuromuscular weakness despite receiving full supportive care, which may include physical therapy [1-6]. In patients requiring mechanical

*Correspondence: carol.hodgson@monash.edu

'Australia and New Zealand Intensive Care Research Centre, School of Public

Health \& Preventive Medicine, Monash University, The Alfred Centre, 99

Commercial Road, Melbourne, VIC 3004, Australia

Full list of author information is available at the end of the article ventilation for longer than 7 days, the incidence of ICUacquired (neuromuscular) weakness is reported to be between 25 and $60 \%$ (Table 1) [1,7,8]. Such weakness may contribute to increased duration of mechanical ventilation, increased length of stay in the ICU and hospital, and poor quality of life among survivors [9-11]. These data suggest that any interventions which may attenuate such weakness and/or shorten the duration of recovery have the potential to improve both the quality of life of patients and reduce healthcare costs. Early mobilization (EM) may represent one such intervention.

In general terms, EM of ICU patients includes the application of traditional modes of physical therapy at an earlier stage than and delivered more regularly than conventional practice, and/or the early use of novel mobilization techniques (for example, cycle ergometry, transcutaneous electrical muscle stimulation). EM appears physiologically logical in patients who would otherwise remain almost immobile, and may also be a safe and feasible process. More importantly, EM may also improve functional recovery, reduce the ICU length of stay, decrease readmissions to the ICU and even improve survival [12-16]. Yet limited systematic attention and analysis has so far been applied to the understanding and assessment of EM [17]. In this article we aim to define the concept of EM in comparison with traditional physical therapy, to review the evidence for its feasibility, safety and possible efficacy, and to define the research agenda for its more comprehensive assessment.

\section{Traditional physical therapy}

There are international guidelines on the traditional approach to physical therapy for patients in the ICU. They include the application of a passive range of movements and the encouragement of an active range of movements early in the ICU stay [18]. Attempts at full active mobilization are often reserved until after the acute phase of the illness has resolved. In particular, it is recognized that rehabilitation may not commence until after ICU discharge, as the patients are viewed as too sick to participate whilst receiving mechanical ventilation. These traditional practices are not based on high-quality evidence and are simply derived from expert opinion. Despite such opinions, however, practice and attitudes surrounding physical therapy and mobilization in the 


\section{Table 1. Diagnostic criteria for ICU-acquired weakness}

Weakness associated with critical illness

Weakness is bilateral, flaccid and involves both proximal and distal muscles but generally spares the cranial nerves

Medical Research Council sum score $<48$

Prolonged mechanical ventilation

Other causes of weakness have been excluded

ICU show wide variability worldwide [19], and even within the same country [20].

The evidence to support the use of passive movements as part of a program of early mobilization is weak [16]. Such evidence suggests that passive movements may prevent protein degradation, maintain muscle mass and alter the inflammatory profile in humans [21,22]. For example, in 20 subjects with severe sepsis or septic shock randomized to 30 minutes of predominantly passive exercise or no intervention, the passive exercise group preserved fat-free mass, decreased IL- 6 and increased IL-10 levels compared with control patients who lost $7.2 \%$ of fat free mass in the first 7 days following admission to the ICU [23]. Clearly this level of evidence is minimal and requires further investigation. Clinical observation, however, suggests that more than simple passive movement should be done in order to help preserve muscle strength. EM might represent a better approach than traditional delayed passive movements. Before such an intervention can be advocated, however, it needs to be defined.

\section{What is early mobilization?}

EM is the intensification and early application (within the first 2 to 5 days of critical illness) of the physical therapy that is administered to critically ill patients (Table 2). EM may also include additional specific mobilization-enhancing interventions such as active mobilization of patients requiring mechanical ventilation and the use of novel techniques such as cycle ergometry and transcutaneous electrical muscle stimulation (TEMS). In the ICU, EM is applied with the intention of maintaining or restoring musculoskeletal strength and function, thereby potentially improving functional, patient-centered outcomes. A major limitation in the ability to determine the outcomes following EM is the variety of different techniques employed, and the lack of standardization and definition of them across studies (Table 2).

Two randomized, controlled, clinical trials [12,24] and several observational studies [4,25-31] provide data on the feasibility and safety of EM as well as preliminary data on its efficacy in patients dependent on ventilatory support (Table 2). For instance, in an observational study, Bailey and colleagues described 1,449 EM interventions in 103 patients [25]. Overall, 53\% of these interventions included ambulating patients that were dependent on positive pressure ventilation via an endotracheal tube or tracheostomy. Only 1\% of these EM activities were associated with an adverse event. These events included five episodes of the patient falling to their knees without injury, three episodes of hypotension to a systolic blood pressure $<90 \mathrm{mmHg}$, one case of increase in systolic blood pressure to $>200 \mathrm{mmHg}$, three episodes of oxygen saturation decreases to $<80 \%$ and the removal of one enteral feeding tube. This type of EM treatment was resourced from within the existing ICU staff structure, including ICU nurses, technicians, physical therapists and respiratory therapists.

In a further study, the same group described a beforeand-after cohort study in 104 patients with respiratory failure who were transferred from another ICU to their respiratory ICU [28]. Transfer to the EM-based respiratory ICU increased the probability of ambulation $(P<0.0001)$ during the patient's ICU stay. By multivariate logistic regression analysis, independent predictors of increased ambulation were transfer to the respiratory ICU with a commitment to EM, female gender, absence of sedatives and lower Acute Physiology and Chronic Health Evaluation II scores. Eighty-eight percent of patients survived to hospital discharge with a mean ambulated distance in the ICU of 200 feet.

In addition to the above work, Schweickert and colleagues completed a prospective, outcome assessorblinded, randomized trial of EM and occupational therapy in two centers in the USA [12]. In this study, patients who were mechanically ventilated for $<72$ hours and expected to stay ventilated in the next 24 hours were randomized either to an EM protocol (rapid progression from passive range of movements to active range of movements, to bed mobility, to sitting balance, to standing, to standing transfers and gait re-education during sedation interruption) or to a control group, which underwent physical and occupational therapy as prescribed by standard care, typically only after extubation. This trial found that EM was safe and feasible and that it was associated with improved functional outcomes as measured using the Katz Index [32] and independent walking at hospital discharge. Importantly, patients in the EM intervention group started physical therapy earlier (1.5 days vs. 7.3 days, $P=0.0001$ ) and were significantly more likely to return to functional independence (defined as being able to wash, dress, groom, eat, transfer from bed to chair and walk independently) at hospital discharge (59\% vs. $35 \%, P=0.02$ ). This study differed from other trials because patients were mobilized very early (day 1.5 on average) and the results documented functional outcomes in a blinded manner. Major adverse events were rare (one in 498 EM-related events, with no extubations, falls or change in systolic blood pressure and one episode 
Table 2. Observational studies of early mobilization in the ICU

\begin{tabular}{|c|c|c|c|c|}
\hline Study & $\begin{array}{l}\text { Number of } \\
\text { patients }\end{array}$ & Inclusion & Intervention & Primary outcome and key findings \\
\hline Bailey and colleagues [25] & 103 & $\begin{array}{l}\text { Acute respiratory failure } \\
\text { with } \mathrm{MV}>4 \text { days }\end{array}$ & Sit on bed, sit on chair and ambulate & $\begin{array}{l}\text { Early activity events: } 1,449 \text { ( } 53 \% \text { ambulate). } \\
\text { Adverse events: }<1 \% \text { (fall to the knees with } \\
\text { no injury, SBP }>200 \text { or }<90 \text { mm Hg and } \\
\text { desaturation }<80 \% \text { ) }\end{array}$ \\
\hline $\begin{array}{l}\text { Thomsen and colleagues } \\
\text { [28] }\end{array}$ & 104 & $\begin{array}{l}\text { Acute respiratory failure } \\
\text { with } M V>4 \text { days }\end{array}$ & $\begin{array}{l}\text { Early activity protocol; PROM, SOEOB, } \\
\text { transfer to chair, walk }\end{array}$ & Ambulation (increased probability $P<0.0001$ ) \\
\hline Morris and colleagues [27] & 165 & $\begin{array}{l}\text { Medical patients with } \\
\text { acute respiratory failure } \\
\text { requiring MV }\end{array}$ & $\begin{array}{l}\text { Early activity protocol with four levels of } \\
\text { activity: PROM, active resisted exercise } \\
\text { and sitting, SOEOB, and transfer to chair }\end{array}$ & $\begin{array}{l}\text { PT (more patients in the protocol group } \\
\text { received PT versus usual care, } 80 \% \text { vs. } 47 \% \text {, } \\
P \leq 0.001 \text { ) }\end{array}$ \\
\hline Zanni and colleagues [29] & 19 & $\begin{array}{l}\text { Medical patients } \\
\text { ventilated }>4 \text { days }\end{array}$ & $\begin{array}{l}\text { Individualized stretching, strengthening, } \\
\text { balance training and functional activities } \\
\text { (rolling, sitting, standing, walking, } \\
\text { grooming, bathing) }\end{array}$ & $\begin{array}{l}\text { Total consultations to PT and OT per patient: } \\
\text { median } 2 \text { ( } 1 \text { to } 4 \text { ). Duration of rehabilitation } \\
\text { (minutes): median } 45 \text { ( } 34 \text { to } 47 \text { ) }\end{array}$ \\
\hline $\begin{array}{l}\text { Needham and colleagues } \\
\text { [4] }\end{array}$ & 57 & $\begin{array}{l}\text { Medical patients } \\
\text { ventilated }>4 \text { days }\end{array}$ & $\begin{array}{l}\text { Multidiscplinary team to focus on } \\
\text { decreased sedation and increased PT } \\
\text { and OT, particularly with functional } \\
\text { mobility }\end{array}$ & $\begin{array}{l}\text { Sedation (benzodiazepam reduced } \\
P<0.002 \text { ). Rehabilitation treatments } \\
\text { (increased } P<0.001 \text { ). Functional mobility } \\
\text { (treatment involving sitting or greater } \\
\text { increased } P=0.03 \text { ) }\end{array}$ \\
\hline $\begin{array}{l}\text { Bourdin and colleagues } \\
\text { [26] }\end{array}$ & 20 & $\begin{array}{l}\text { Medical patients in ICU } \\
\geq 7 \text { days and } M V \geq 2 \text { days }\end{array}$ & Chair sitting, tilt table and walking & $\begin{array}{l}\text { Physiological response: HR and RR } \\
\text { increased with sitting, tilting up with arms } \\
\text { unsupported and walking, oxygen saturation } \\
\text { decreased with tilting up arms unsupported } \\
\text { and walking }\end{array}$ \\
\hline Kho and colleagues [51] & 22 & $\begin{array}{l}\text { Medical ICU adults } \\
\text { receiving PT }\end{array}$ & Video games & $\begin{array}{l}\text { Safety (zero adverse events). Feasibility (5\% } \\
\text { patients receiving PT used video games) }\end{array}$ \\
\hline Genc and colleagues [57] & 31 & $\begin{array}{l}\text { Critically ill obese } \\
\text { patients }\end{array}$ & $\begin{array}{l}\text { Mobilization; SOEOB, standing, transfer } \\
\text { to chair by walking, sitting in the chair }\end{array}$ & $\begin{array}{l}\text { Transient episodes of altered SBP or HR } \\
\text { in six patients. No deterioration in clinical } \\
\text { status. } \mathrm{SpO}_{2} \text { significantly increased after } \\
\text { mobilization }\end{array}$ \\
\hline $\begin{array}{l}\text { Leditschke and colleagues } \\
\text { [58] }\end{array}$ & 106 & $\begin{array}{l}\text { Mixed medical-surgical } \\
\text { ICU }\end{array}$ & $\begin{array}{l}\text { Active mobilization: MOS > } 30 \text { seconds. } \\
\text { Active transfer: transfer bed-chair } \\
\text { against gravity. Passive transfer: passively } \\
\text { lifted to out of bed (lifter, sling) }\end{array}$ & $\begin{array}{l}\text { Two adverse events in } 176 \text { mobilization } \\
\text { episodes (1.1\%), which were hypotension } \\
\text { requiring return to bed and fluid loading } \\
\text { or vasopressors. Avoidable barriers to } \\
\text { mobilization include femoral lines, sedation } \\
\text { and scheduling procedures }\end{array}$ \\
\hline
\end{tabular}

$\mathrm{HR}$, heart rate; MOS, marching on the spot; MV, mechanical ventilation; OT, occupational therapy; PROM, passive range of movement; PT, physical therapy; RR, respiratory rate; $\mathrm{SBP}$, systolic blood pressure; $\mathrm{SOEOB}$, sit over edge of bed; $\mathrm{SpO}_{2}$, oxygen saturation measured by pulse oximetry.

of decreased oxygen saturation $<80 \%)$. However, in order to maximize the degree of mobilization, new techniques are rapidly emerging that can be more easily and perhaps more safely applied to ventilated supine patients.

\section{Early mobilization using novel techniques Cycle ergometer}

A cycle ergometer is a stationary cycle with an automatic mechanism that can alter the amount of work performed by the patient. The cycle can be used passively (no work from the patient) or actively (Figure 1). Cycle ergometry has been tested in healthy subjects as part of the space research program and has been found to preserve thigh muscle thickness during prolonged immobilization [33]. The method has also been shown to be safe and feasible in studies during hemodialysis [34] and in patients with chronic obstructive pulmonary disease [35].
Cycle ergometer-based mobilization in addition to standard care has now been used as a form of EM in a single-center randomized trial of 90 critically ill patients, and compared with standard care alone. In this study, cycle ergometer-based mobilization improved the median 6-minute walk distance at hospital discharge (196 m vs. $143 \mathrm{~m}, P<0.05)$ [24]. In addition, the mobilization method was reported to be safe and feasible, with a median of four cycle sessions completed per week and the time taken from ergometer set-up to clean-up inclusive reported at 30 to 40 minutes. There were no major adverse events and only $4 \%$ of cycle sessions were stopped early due to adverse changes in oxygen saturation $<90 \%$.

\section{Transcutaneous electrical muscle stimulation}

TEMS has been used to preserve muscle mass and strength in patients with chronic heart failure [36,37] and 


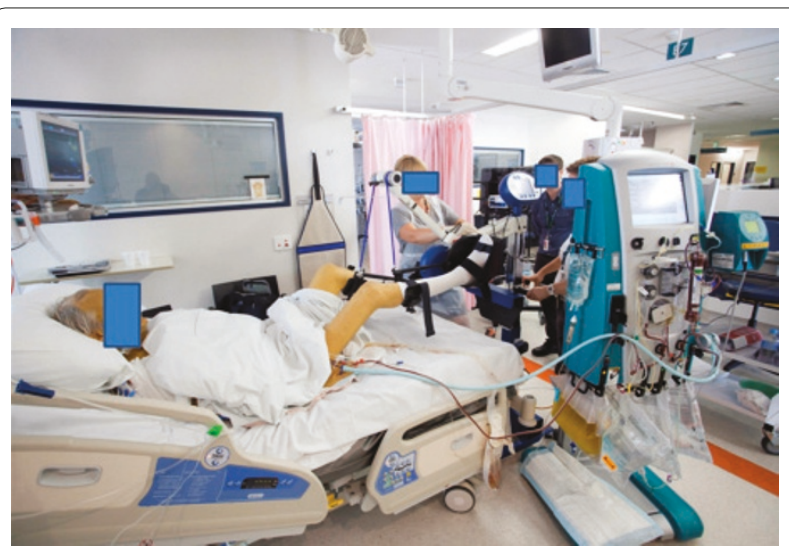

Figure 1. A ventilated patient using a cycle ergometer in the ICU.

in patients with chronic obstructive pulmonary disease [38]. In a recent systematic review, TEMS was found to improve muscle strength, exercise capacity and diseasespecific health status [39]. TEMS is of particular interest in the ICU setting because the loss of muscle mass is rapid and more severe than in other chronic conditions [40]. In addition, the TEMS technique can be used easily in immobile sedated patients.

Despite the physiological attractiveness and promise of TEMS, the randomized controlled trials that have evaluated the effects of EM by means of TEMS initiated in the first 7 days of ICU stay have reported conflicting results [41-45]. Differences in patient selection, the inclusion or exclusion of patients with sepsis, the application of TEMS to heterogeneous populations, and variable study methodology have all probably contributed to discrepancies in reported outcomes.

The largest study of TEMS to date investigated 140 critically ill patients and randomly assigned them to TEMS or standard care [44]. TEMS was conducted daily for 55 minutes to the lower limb (vastus lateralis, vastus medialis and peroneous longus muscles). The primary outcome was ICU-acquired (neuromuscular) weakness diagnosed using the Medical Research Council score $(<48 / 60)$ by two unblinded independent investigators. The Medical Research Council score was significantly higher in patients in the TEMS group compared with those of the control group (58 (33 to 60) vs. 52 (2 to 60)). However, this study has been criticized for several reasons [46]. First, measurement of the primary outcome could only be performed in awake, cooperative patients. This limitation excluded 39 patients who died and 44 patients who were cognitively impaired from the final analysis. Accordingly, the intention-to-treat principle was violated. In the intervention group, data from three patients were also excluded due to the use of neuromuscular blockers. Finally, TEMS was applied only to the lower limb but the Medical Research Council score reflects upper and lower limb strength. Although it is theoretically possible that TEMS has systemic effects, the change in upper limb strength seems unusual [41]. There was no report of patient tolerance to TEMS. Future studies should include a report of patient discomfort with the use of this technique.

Other small randomized controlled trials ( $n<25$ subjects) have evaluated the effects of TEMS in patients who were chronically critically ill and requiring mechanical ventilation for $>14$ days. Such trials have reported improvements in muscle mass as measured by ultrasound [47], muscle strength as assessed using manual muscle testing ( $2.2 \pm 1.0$ vs. $1.3 \pm 0.8, P=0.02)$ and function as measured by changes in the number of days required to transfer from bed to chair ( $11 \pm 2$ days vs. $14 \pm 2$ days, $P=0.001)$ [48]. In this regard, the results of the small study $(n=24)$ by Zanotti and colleagues are of particular interest because improvements in muscle strength were accompanied by improved function [48]. The intervention protocol, however, included the use of TEMS in conjunction with a program of active limb exercise in ventilator-dependent patients with chronic obstructive pulmonary disease. Their results therefore suggest that TEMS may act synergistically with active exercise and thus should not be used in isolation but should rather be a useful component of a wider-ranging EM protocol aimed at restoring muscle mass in chronically critically ill patients. There was no report of patient tolerance to TEMS.

Despite the above reports, the assessment of efficacy in small trials remains difficult and investigators are increasingly focusing on surrogate outcomes that would justify the conduct of larger phase II studies. Among such outcomes, muscle layer thickness and muscle crosssectional area measured by ultrasound appear to have a relationship with muscle strength [49,50]. Further research is required to establish whether these outcomes are associated with sustained improvement in function and health-related quality of life and can be reliably used as surrogates for such clinical outcomes.

\section{Custom-made technological aids}

Ambulation is a specific EM technique that is used in the ICU to improve functional recovery $[25,27,29]$. Standard hospital equipment may generally be adequate. When ambulating a mechanically ventilated patient, however, the standard equipment may not maximize safety and effectiveness [40]. For example, some hospitals have had their own engineers design a custom-made walker for ventilated patients that incorporates a walking frame on wheels, an intravenous pole, an oxygen basket and a platform to support a ventilator, all in a single device (Figure 2). This type of equipment may improve the safety of the patient as the nurse and physical therapist have 
their hands free of equipment and are able to concentrate on the patient's gait, balance and physiological response to exercise, such as their respiratory rate. There is no evidence that patients require electrocardiogram monitoring during early mobilization.

\section{Video therapies}

Among other descriptive papers of novel techniques for EM in the ICU, the feasibility of Wii and other interactive video therapies has been described. In an observational, single-center study, Kho and colleagues investigated the use of video therapies as a form of EM in critical illness [51]. Of 410 patients receiving physical therapy, 5\% used video games for balance (52\%) and endurance (45\%). The most common games were boxing, bowling and balance board. No adverse events occurred (95\% upper confidence limit for safety event rate: $8.4 \%$ ). No trials, however, have compared such interventions with a control group receiving standard care.

\section{Barriers to early mobilization}

Although EM seems intuitively useful and physiologically logical it can in fact be a complex and effort-intensive therapy, which is made even more challenging by the presence of multiple barriers that impede its wider uptake [30]. Such barriers include inadequate staff to deliver physical therapy, lack of equipment, concern regarding patient safety and physiological stability [31], sedation and ventilation practices, placement of vascular lines, and the paucity of data on efficacy and healtheconomic evaluation to convince clinicians to apply EM [14].

A key barrier to the delivery of EM is concern about the safety of the patient [14]. Adverse events may include the dislodgement of vascular lines, nasogastric tubes and urinary catheters and, much more importantly, of an artificial airway, leading to life-threatening hypoxia. To counter these concerns, however, there is an emerging body of data suggesting that EM does not impose an increased risk to patients if it is performed with appropriately trained staff $[4,12,24,25,27,28,30]$. In several studies conducted in US centers, EM involved a mobilization team of three ICU clinicians, including a physical therapist, a nurse and an occupational therapist or an assistant $[25,27,28]$. In addition, patients were carefully evaluated holistically prior to undertaking EM with a comprehensive assessment of age, level of fitness prior to ICU admission, presenting condition, tolerance of other interventions and the amount of ventilatory and cardiac support required prior to EM.

EM is feasible only if the patient is awake and cooperative [52] and therefore the use of sedation needs to be minimized to facilitate EM [53]. The importance of interactions between the degree of sedation and the

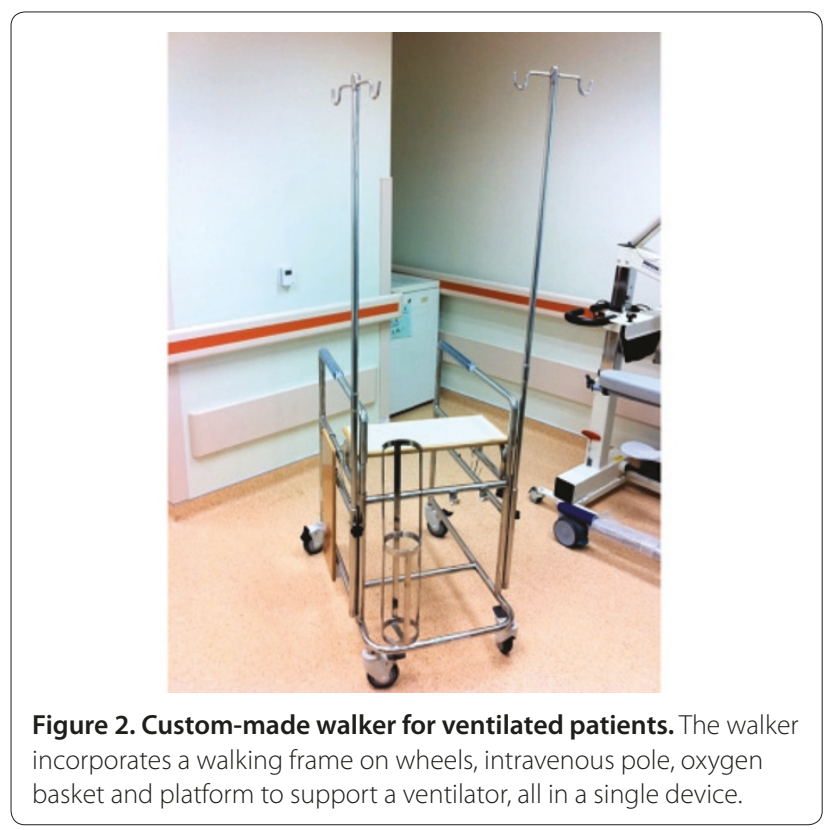

ability to apply EM has been highlighted in several publications [30,53]. Other key factors that appear to be associated with successful EM include adequate pain management and early recognition and management of delirium [13,54-56].

An additional consideration is the availability of sufficient staff and equipment to facilitate EM. Physical therapists should be an integral part of the interdisciplinary team in the ICU involved in the implementation of EM. Having at least one ICU nurse available is also important (Figure 3). In the USA, a respiratory therapist would also be included to disconnect the ventilator and assist with a portable ventilator [15]. The need for such complex multiskilled personnel with sufficient training in EM and the need for appropriate equipment may be the major barrier to the implementation of EM in most ICUs worldwide.

\section{Early mobilization research agenda}

International differences in staff availability result in heterogeneous research questions in relation to EM in different countries. In American studies, for example, research has concentrated on providing information to justify appropriate resources for physical therapy input in ICUs [4]. In contrast, in Europe and Australia physical therapy is generally considered part of standard management. Two national surveys, however, have reported a striking degree of variability between institutions within the same country in terms of referral to physical therapy during critical illness, staff ratios and frequency of such therapy $[19,20]$. This variability in practice underscores the importance of carefully defining and understanding usual care prior to undertaking any interventional studies 


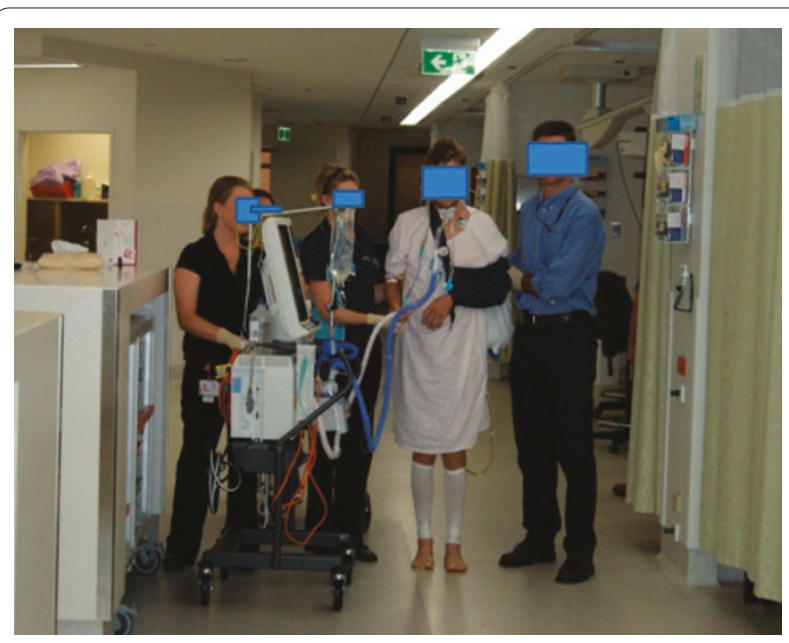

Figure 3. A ventilated patient walking with assistance of physical therapists and a trolley.

to evaluate the efficacy and safety of EM in different jurisdictions. The variability also highlights the limited external validity of single-center studies.

\section{Key research questions}

Standard mobilization practice in ICUs nationally and internationally remains poorly defined. Until standard mobilization is clearly defined and measured in multicenter studies it is impossible to conduct studies of any EM interventions that are relevant to modern ICU treatment, have external validity, and provide sufficient treatment separation. There may be limitations to establishing a true, ethical control group to establish the effect of EM, especially in those countries where physiotherapy practice already includes elements of EM. The typical functional outcomes of candidate patients who survive and are treated with standard care also need to be defined. Once baseline practice and functional outcomes are established in multicenter prospective cohort studies, then multicenter pilot studies of a candidate EM intervention can be tested for separation and contamination. In addition, several potential relevant outcomes (muscle thickness, muscle strength for different muscle groups, functional independence) must also be assessed as outcome measures for EM interventions and their relationships with each other understood in order to power future larger studies of these interventions.

\section{Conclusion}

ICU survivors recovering from a prolonged illness often have muscle weakness and major functional impairment. Early mobilization is a physiologically logical candidate intervention to attenuate such weakness. Observational studies and initial small randomized trials evaluating EM suggest safety and feasibility. These studies also suggest that EM has the potential to improve functional outcomes in survivors. Unfortunately, such studies are mostly single center in design and carry limited external validity. Further trials to investigate the potential benefits of EM and the best techniques to maximize its efficacy are warranted but require careful consideration of standard practice, optimal treatment strategies and outcome measures.

\section{Abbreviations \\ EM, early mobilization; IL, interleukin; TEMS, transcutaneous electrical muscle stimulation.}

\section{Competing interests}

The authors declare that they have no competing interests.

\section{Acknowedgements}

Written consent for publication was obtained by the patients.

\section{Author details}

${ }^{1}$ Australia and New Zealand Intensive Care Research Centre, School of Public Health \& Preventive Medicine, Monash University, The Alfred Centre, 99 Commercial Road, Melbourne, VIC, Australia, 3004. ${ }^{2}$ The Alfred, Melbourne, Commercial Rd Prahran, VIC, Australia, 3181. ${ }^{3}$ Austin Health, 145 Studley Rd, Heidelberg, VIC, Australia, 3084. ${ }^{4}$ University of Melbourne, Grattan St Parkville, Melbourne, VIC, Australia, 3010. ${ }^{5}$ Curtin University, GPO Box U1987, Perth, Western Australia, 6845. ${ }^{6}$ Royal Perth Hospital, 197 Wellington St, Perth, WA, Australia, 6000. ${ }^{7}$ The St George Hospital, Gray St, Kogarah, NSW, Australia, 2217. ${ }^{8}$ The University of New South Wales, High St, Kensington, NSW, Australia, 2052. ${ }^{9}$ The George Institute For Global Health, Level 13, 321 Kent Street, Sydney, NSW, Australia, 2000.

Published: 28 February 2012

\section{References}

1. Saxena MK, Hodgson CL: Intensive care unit acquired weakness. Anaesth Intensive Care Med 2012, 13:145-147.

2. Morris PE, Griffin L, Berry M, Thompson C, Hite RD, Winkelman C, Hopkins RO, Ross A, Dixon L, Leach S, Haponik E: Receiving early mobility during an intensive care unit admission is a predictor of improved outcomes in acute respiratory failure. Am J Med Sci 2011, 341:373-377.

3. Needham DM: Mobilizing patients in the intensive care unit: improving neuromuscular weakness and physical function. JAMA 2008, 300:1685-1690.

4. Needham DM, Korupolu R, Zanni JM, Pradhan P, Colantuoni E, Palmer JB, Brower RG, Fan E: Early physical medicine and rehabilitation for patients with acute respiratory failure: a quality improvement project. Arch Phys Med Rehabil 2010, 91:536-542.

5. Griffiths RD, Hall JB: Intensive care unit-acquired weakness. Crit Care Med 2010, 38:779-787.

6. Maramattom BV, Wijdicks EF: Acute neuromuscular weakness in the intensive care unit. Crit Care Med 2006, 34:2835-2841.

7. de Jonghe B, Lacherade JC, Sharshar T, Outin H: Intensive care unit-acquired weakness: risk factors and prevention. Crit Care Med 2009, 37(10 Suppl):S309-S315.

8. Nordon-Craft A, Moss M, Quan D, Schenkman M: Intensive care unitacquired weakness. J Neuro Phys Ther 2011, 35:133-140.

9. Herridge MS: Long-term outcomes after critical illness: past, present, future. Curr Opin Crit Care 2007, 13:473-475.

10. Herridge MS: Legacy of intensive care unit-acquired weakness. Crit Care Med 2009, 37(10 Suppl):S457-S461.

11. Rubenfeld GD, Herridge MS: Epidemiology and outcomes of acute lung injury. Chest 2007, 131:554-562.

12. Schweickert WD, Pohlman MC, Pohlman AS, Nigos C, Pawlik AJ, Esbrook CL, Spears L, Miller M, Franczyk M, Deprizio D, Schmidt GA,Bowman A, Barr R, McCallister KE, Hall JB, Kress JP: Early physical and occupational therapy in mechanically ventilated, critically ill patients: a randomised controlled trial. Lancet 2009, 373:1874-1882.

13. Morandi A, Brummel NE, Ely EW: Sedation, delirium and mechanical ventilation: the 'ABCDE' approach. Curr Opin Crit Care 2011, 17:43-49. 
14. Morris PE: Moving our critically ill patients: mobility barriers and benefits. Crit Care Clin 2007, 23:1-20

15. Perme C, Chandrashekar R: Early mobility and walking program for patients in intensive care units: creating a standard of care. Am J Crit Care 2009, 18:212-221.

16. O'Connor ED, Walsham J: Should we mobilise critically ill patients? A review. Crit Care Resusc 2009, 11:290-300.

17. Adler J, Malone D: Early mobilization in the intensive care unit: a systematic review. Cardiopulm Phys Ther J 2012, 23:5-13.

18. Gosselink R, Bott J, Johnson M, Dean E, Nava S, Norrenberg M, Schonhofer B, Stiller $K$, van de Leur $H$, Vincent JL: Physiotherapy for adult patients with critical illness: recommendations of the European Respiratory Society and European Society of Intensive Care Medicine Task Force on Physiotherapy for Critically III Patients. Intensive Care Med 2008, 34:1188-1199.

19. Norrenberg M, Vincent JL: A profile of European intensive care unit physiotherapists. European Society of Intensive Care Medicine. Intensive Care Med 2000, 26:988-994.

20. Hodgin KE, Nordon-Craft A, McFann KK, Mealer ML, Moss M: Physical therapy utilization in intensive care units: results from a national survey. Crit Care Med 2009, 37:561-568

21. Griffiths RD, Palmer TE, Helliwell T, P. M, MacMillan RR: Effect of passive stretching on the wasting of muscle in the critically ill. Nutrition 1995 1:428-432.

22. Paratz JD, Kayambu G: Early exercise and attenuation of myopathy in patient with sepsis in ICU. Phys Ther Rev 2011, 16:58-65.

23. Kayambu G, Boots RJ, Paratz JD: Early rehabilitation in sepsis: a prospective randomised controlled trial investigating functional and physiological outcomes The i-PERFORM Trial (Protocol Article). BMC Anesthesiol 2011, $11: 21$.

24. Burtin C, Clerckx B, Robbeets C, Ferdinande P, Langer D, Troosters T, Hermans G, Decramer M, Gosselink R: Early exercise in critically ill patients enhances short-term functional recovery. Crit Care Med 2009, 37:2499-2505.

25. Bailey P, Thomsen GE, Spuhler VJ, Blair R, Jewkes J, Bezdjian L, Veale K, Rodriquez L, Hopkins RO: Early activity is feasible and safe in respiratory failure patients. Crit Care Med 2007, 35:139-145.

26. Bourdin G, Barbier J, Burle JF, Durante G, Passant S, Vincent B, Badet M, Bayle F, Richard JC, Guerin C: The feasibility of early physical activity in intensive care unit patients: a prospective observational one-center study. Respir Care 2010, 55:400-407

27. Morris PE, Goad A, Thompson C, Taylor K, Harry B, Passmore L, Ross A, Anderson L, Baker S, Sanchez M, Penley L, Howard A, Dixon L, Leach S, Small R, Hite RD, Haponik E: Early intensive care unit mobility therapy in the treatment of acute respiratory failure. Crit Care Med 2008, 36:2238-2243.

28. Thomsen GE, Snow GL, Rodriguez L, Hopkins RO: Patients with respiratory failure increase ambulation after transfer to an intensive care unit where early activity is a priority. Crit Care Med 2008, 36:1119-1124.

29. Zanni JM, Korupolu R, Fan E, Pradhan P, Janjua K, Palmer JB, Brower RG, Needham DM: Rehabilitation therapy and outcomes in acute respiratory failure: an observational pilot project. J Crit Care 2010, 25:254-262.

30. Leditschke A, Green M, Irvine J, Bissett B, Mitchell I: What are the barriers to mobilizing intensive care patients? Cardiopulm Phys Ther J 2012, 23:26-29.

31. Genc A, Ozyurek S, Koca U, Gunerli A: Respiratory and hemodynamic responses to mobilzation of critically ill obese patients. Cardiopulm Phys Ther J 2012, 23:14-18

32. Brorsson B, Asberg KH: Katz index of independence in ADL. Reliability and validity in short-term care. Scand J Rehabil Med 1984, 16:125-132.

33. Ellis S, Kirby LC, Greenleaf JE: Lower extremity muscle thickness during 30-day 6 degrees head-down bed rest with isotonic and isokinetic exercise training. Aviat Space Environ Med 1993, 64:1011-1015.

34. Moug SJ, Grant S, Creed G, Boulton Jones M: Exercise during haemodialysis: West of Scotland pilot study. Scott Med J 2004, 49:14-17.

35. Larson JL, Covey MK, Wirtz SE, Berry JK, Alex CG, Langbein WE, Edwards L: Cycle ergometer and inspiratory muscle training in chronic obstructive pulmonary disease. Am J Respir Crit Care Med 1999, 160:500-507.

36. Sbruzzi G, Schaan BD, Pimentel GL, Signori LU, Da Silva AN, Oshiro MS, Irigoyen MC, Plentz RD: Effects of low frequency functional electrical stimulation with 15 and $50 \mathrm{~Hz}$ on muscle strength in heart failure patients. Disabil Rehabil 2011, 33:486-493.

37. Quittan M, Wiesinger GF, Sturm B, Puig S, Mayr W, Sochor A, Paternostro T, Resch KL, Pacher R, Fialka-Moser V: Improvement of thigh muscles by neuromuscular electrical stimulation in patients with refractory heart failure: a single-blind, randomized, controlled trial. Am J Phys Med Rehabil 2001, 80:206-214.

38. Neder JA, Sword D, Ward SA, Mackay E, Cochrane LM, Clark CJ: Home based neuromuscular electrical stimulation as a new rehabilitative strategy for severely disabled patients with chronic obstructive pulmonary disease (COPD). Thorax 2001, 57:33-37.

39. Sillen MJ, Speksnijder CM, Eterman RM, Janssen PP, Wagers SS, Wouters EF, Uszko-Lencer NH, Spruit MA: Effects of neuromuscular electrical stimulation of muscles of ambulation in patients with chronic heart failure or COPD: a systematic review of the English-language literature. Chest 2009, 136:44-61.

40. Needham DM, Truong AD, Fan E: Technology to enhance physical rehabilitation of critically ill patients. Crit Care Med 2009, 37(10 Suppl):S436-S441.

41. Ali NA: Have we found the prevention for intensive care unit-acquired paresis? Crit Care 2010, 14:160

42. Gerovasili V, Stefanidis K, Vitzilaios K, Karatzanos E, Politis P, Koroneos A Chatzimichail A, Routsi C, Roussos C, Nanas S: Electrical muscle stimulation preserves the muscle mass of critically ill patients: a randomized study. Crit Care 2009, 13:R161.

43. Gerovasili V, Tripodaki E, Karatzanos E, Pitsolis T, Markaki V, Zervakis D, Routsi C, Roussos C, Nanas S: Short-term systemic effect of electrical muscle stimulation in critically ill patients. Chest 2009, 136:1249-1256.

44. Routsi C, Gerovasili V, Vasileiadis I, Karatzanos E, Pitsolis T, Tripodaki E, Markaki V, Zervakis D, Nanas S: Electrical muscle stimulation prevents critical illness polyneuromyopathy: a randomized parallel intervention trial. Crit Care 2010, $14 R 74$.

45. Rodriguez PO, Setten M, Maskin LP, Bonelli I, Vidomlansky SR, Attie S, Frosiani SL, Kozima S, Valentini R: Muscle weakness in septic patients requiring mechanical ventilation: protective effect of transcutaneous neuromuscular electrical stimulation. J Crit Care 2012, 27:e311-e318.

46. Rodriguez PO, Setten M, Valentini R: Electrical muscle stimulation for prevention of critical illness polyneuropathy. Crit Care 2010, 14:428.

47. Gruther W, Kainberger F, Fialka-Moser V, Paternostro-Sluga T, Quittan M, Spiss C, Crevenna R: Effects of neuromuscular electrical stimulation on muscle layer thickness of knee extensor muscles in intensive care unit patients: a pilot study. J Rehabil Med 2010, 42:593-597.

48. Zanotti E, Felicetti G, Maini M, Fracchia C: Peripheral muscle strength training in bed-bound patients with COPD receiving mechanical ventilation. Chest 2003, 124:292-296.

49. Chi-Fishman G, Hicks JE, Cintas HM, Sonies BC, Gerber LH: Ultrasound imaging distinguishes between normal and weak muscle. Arch Phys Med Rehabil 2004, 85:980-986.

50. Seymour JM, Ward K, Sidhu PS, Puthucheary Z, Steier J, Jolley CJ, Rafferty G, Polkey Ml, Moxham J: Ultrasound measurement of rectus femoris crosssectional area and the relationship with quadriceps strength in COPD. Thorax 2009, 64:418-423.

51. Kho ME, Damluji A, Zanni JM, Needham DM: Feasibility and observed safety of interactive video games for physical rehabilitation in the intensive care unit: a case series. J Crit Care 2012, 27:e211-e216.

52. Hopkins RO, Spuhler VJ: Strategies for promoting early activity in critically ill mechanically ventilated patients. AACN Adv Crit Care 2009, 20:277-289.

53. Vasilevskis EE, Ely EW, Speroff T, Pun BT, Boehm L, Dittus RS: Reducing iatrogenic risks: ICU-acquired delirium and weakness - crossing the quality chasm. Chest 2010, 138:1224-1233.

54. Bigatello LM, Citerio G: The long-lasting damage of delirium: another burden to intensive care unit survivors. Crit Care Med 2012, 40:319-320.

55. Morandi A, Jackson JC: Delirium in the intensive care unit: a review. Neurol Clin 2011, 29:749-763

56. Page V, Gough K: Management of delirium in the intensive care unit. $\mathrm{Br} J$ Hosp Med (Lond) 2010, 71:372-376.

57. Genc A, Ozyurek S, Koca U, Gunerli A: Respiratory and hemodynamic responses to mobilization of critically ill obese patients. Cardiopulm Phys Ther J 2012, 23:14-18.

58. Leditschke IA, Green M, Irvine J, Bissett B, Mitchell IA: What are the barriers to mobilizing intensive care patients? Cardiopulm Phys Ther J 2012, 23:26-29.

doi:10.1186/cc11820

Cite this article as: Hodgson $\mathrm{CL}$, et al:: Clinical review: Early patient

mobilization in the ICU. Critical Care 2013, 17:207. 